\title{
GRB 070724B: the first gamma ray burst localized by SuperAGILE and its Swift X-ray afterglow ${ }^{\star}$
}

\author{
E. Del Monte ${ }^{1}$, M. Feroci ${ }^{1}$, L. Pacciani ${ }^{1}$, Y. Evangelista ${ }^{1,2}$, I. Donnarumma ${ }^{1}$, P. Soffitta ${ }^{1}$, E. Costa ${ }^{1}$, I. Lapshov ${ }^{1}$, \\ F. Lazzarotto ${ }^{1}$, M. Rapisarda ${ }^{3}$, A. Argan ${ }^{1}$, G. Barbiellini ${ }^{4,5}$, M. Basset ${ }^{4}$, A. Bulgarelli ${ }^{6}$, P. Caraveo ${ }^{7}$, A. Chen $^{7}$,
} G. Di Cocco ${ }^{6}$, L. Foggetta ${ }^{4}$, F. Fuschino ${ }^{6}$, M. Galli ${ }^{8}$, F. Gianotti ${ }^{6}$, A. Giuliani ${ }^{7}$, C. Labanti ${ }^{6}$, P. Lipari ${ }^{2}$, F. Longo $^{4,5}$, M. Marisaldi ${ }^{6}$, F. Mauri ${ }^{9}$, S. Mereghetti ${ }^{7}$, A. Morselli ${ }^{10}$, A. Pellizzoni ${ }^{7}$, F. Perotti ${ }^{7}$, P. Picozza ${ }^{10}$, M. Prest $^{11}$, G. Pucella ${ }^{1}$, M. Tavani ${ }^{1,10}$, M. Trifoglio ${ }^{6}$, A. Trois ${ }^{1}$, E. Vallazza ${ }^{4}$, S. Vercellone $^{7}$, V. Vittorini ${ }^{1}$, A. Zambra ${ }^{12}$, P. Romano ${ }^{13,14}$, D. N. Burrows ${ }^{15}$, G. Chincarini ${ }^{13,14}$, N. Gehrels ${ }^{16}$, V. La Parola ${ }^{17}$, P. T. O'Brien ${ }^{18}$, J. P. Osborne ${ }^{18}$, B. Preger ${ }^{19,20}$, C. Pittori ${ }^{19,20}$, L. A. Antonelli ${ }^{19,21}$, F. Verrecchia ${ }^{19,20}$, P. Giommi ${ }^{19,22}$, and L. Salotti ${ }^{22}$

1 INAF IASF Roma, via Fosso del Cavaliere 100, 00133 Roma, Italy e-mail: ettore.delmonte@iasf-roma.inaf.it

2 Dip. di Fisica, Università degli Studi di Roma "La Sapienza", P.le A. Moro 5, 00185 Roma, Italy

3 ENEA UTS Fusione Tecnologie, via E. Fermi 45, 00044 Frascati (Rm), Italy

4 INFN Trieste, Padriciano 99, 34012 Trieste, Italy

5 Dip. di Fisica, Università di Trieste, via Valerio 2, 34127 Trieste, Italy

${ }^{6}$ INAF IASF Bologna, via Gobetti 101, 40129 Bologna, Italy

INAF IASF Milano, via E. Bassini 15, 20133 Milano, Italy

8 ENEA C.R. "E. Clementel", via don Fiammelli 2, 40128 Bologna, Italy

9 INFN Pavia, via Bassi 6, 27100 Pavia, Italy

10 Dip. di Fisica, Università degli Studi di Roma "Tor Vergata", via della Ricerca Scientifica 1, 00133 Roma, Italy

11 Dip. di Fisica e Matematica, Università dell'Insubria, via Valleggio 11, 20100 Como, Italy

12 Consorzio Interuniversitario per la Fisica Spaziale, Viale Settimio Severo 63, 10133 Torino, Italy

13 INAF - Osservatorio Astronomico di Brera, via E. Bianchi 46, 23807 Merate (LC), Italy

14 Università degli Studi di Milano-Bicocca, Piazza delle Scienze 3, 20126 Milano, Italy

15 Department of Astronomy \& Astrophysics, Pennsylvania State University, DNBurrows 525 Davey Lab, University Park, PA 16802, USA

16 NASA/Goddard Space Flight Center, Greenbelt, MD 20771, USA

17 INAF IASF Palermo, via U. La Malfa 153, 90146 Palermo, Italy

18 Department of Physics \& Astronomy, University of Leicester, LE1 7RH, UK

19 ASI Science Data Center, via G. Galilei, 00044 Frascati (Rm), Italy

20 INAF personnel resident at ASI Science Data Center

21 Osservatorio Astronomico di Roma, via di Frascati 33, 00040 Monte Porzio Catone (Rm), Italy

22 Agenzia Spaziale Italiana, Unità Osservazione dell'Universo, Viale Liegi 26, 00198 Roma, Italy

Received 9 October 2007 / Accepted 15 November 2007

\section{ABSTRACT}

GRB 070724B is the first gamma ray burst localized by SuperAGILE, the hard X-ray monitor aboard the AGILE satellite. The coordinates of the event were published $\sim 19 \mathrm{~h}$ after the trigger. The Swift X-Ray Telescope pointed at the SuperAGILE location and detected the X-ray afterglow inside the SuperAGILE error circle. The AGILE gamma-ray Tracker and Minicalorimeter did not detect any significant gamma ray emission associated with GRB 070724B in the MeV and GeV range, neither prompt nor delayed. Searches for the optical afterglow were performed by the Swift UVOT and the Palomar automated 60-inch telescopes, resulting in no significant detection. Similarly, the Very Large Array did not detect any radio afterglow. This is the first GRB event associated with an X-ray afterglow with a firm upper limit in the $100 \mathrm{MeV}-30 \mathrm{GeV}$ energy range.

Key words. gamma rays: bursts - X-rays: individuals: GRB 070724B

\section{Introduction}

The Italian AGILE satellite mission (Tavani et al. 2006) was launched on 23 April 2007 from India to an equatorial orbit at $\sim 550 \mathrm{~km}$ altitude and $2.5^{\circ}$ inclination. The AGILE payload includes two imaging instruments: the Gamma Ray

$\star$ Table 1 is only available in electronic form at http: //www . aanda.org
Imaging Detector (GRID), composed of a Silicon Tracker and a Minicalorimeter (MCAL), sensitive in the $30 \mathrm{MeV}-50 \mathrm{GeV}$ energy band, with $\sim 2.5$ sr field of view (FOV) and 15 arcmin source location accuracy ( $\geq 10 \sigma$ detection), and SuperAGILE (Feroci et al. 2007a), a one-dimensional coded aperture instrument, based on silicon microstrip detectors, operating in the nominal 18-45 keV energy band, with a $2 \times 1 \mathrm{D}$ FOV of $68^{\circ} \times 68^{\circ}$ and $\sim 1-2$ arcmin source location accuracy for 
high $\mathrm{S} / \mathrm{N}$ sources. Although the nominal upper boundary of the SuperAGILE energy band is $45 \mathrm{keV}$, for sources with a hard spectrum like the gamma ray bursts (GRBs) a significant signal may be detected up to about $60 \mathrm{keV}$.

AGILE is expected to detect $\sim 10-15$ GRBs per year in the SuperAGILE hard X-ray band and $\sim 5-10$ in the GRID gamma ray band, only few of which will be localized by SuperAGILE due to its smaller FOV. Approximately 1 GRB per week is currently detected by MCAL in the $350 \mathrm{keV}-2.8 \mathrm{MeV}$ energy range. The three GRBs detected by SuperAGILE during the first four months of nominal operation (mid-July to mid-November is roughly consistent with the expected rate. AGILE is equipped with an on-board GRB detection and localization system, based on the SuperAGILE and MCAL ratemeters (Del Monte et al. 2007; Fuschino et al. 2007). Upon trigger, a SuperAGILE localization of the event is searched for and the position is transmitted to Earth within a timescale of minutes by using the ORBCOMM constellation of telecommunication satellites (Deckett 1993). The commissioning phase of the onboard GRB detection system was not complete at the time of GRB 070724B, which was then identified and localized during ground standard quicklook operations.

In this Letter we report the properties of the first GRB localized by SuperAGILE and of its X-ray afterglow discovered by the X-Ray Telescope (XRT; Burrows et al. 2005a) aboard Swift. Throughout this paper the quoted uncertainties are given at $90 \%$ confidence level for one interesting parameter (i.e., $\Delta \chi^{2}=2.71$ ) and times are referred to the SuperAGILE trigger $T_{0}$ (i.e., $t=$ $T-T_{0}$ ), unless otherwise specified.

\section{Observation of GRB 070724B}

\subsection{Prompt emission}

On 24 July 2007 at 23:25:08 UT (hereafter $T_{0}$ ) a gamma ray burst (GRB 070724B) was detected by SuperAGILE. Due to the unavailability of the SuperAGILE on-board GRB detection and localization system and to a problem that delayed the telemetry data transmission, the data processing could only start on ground only $\sim 13 \mathrm{~h}$ after the event. In addition, the mission was still in its early phase, with the calibration of the SuperAGILE astrometry mostly relying on the ground measurements and on a sample of just three known X-ray sources detected in flight.

The first SuperAGILE GRB localization was derived by intersecting the two one-dimensional images shown in Fig. 1, as $\mathrm{RA}=01^{\mathrm{h}} 10^{\mathrm{m}} 31^{\mathrm{s}} .0$, Dec $=+57^{\circ} 40^{\prime} 23^{\prime \prime}$ (equinox 2000) with an uncertainty of 20 arcmin radius (Feroci et al. 2007b), mostly accounted for by the poor calibration of the absolute source positioning available at the epoch of this detection. During the science verification phase the SuperAGILE astrometry is calibrated by means of a raster scan with the Crab Nebula and with the detection of several other sources. With the status of calibration available at the time of writing we derive the GRB refined location as $\mathrm{RA}=01^{\mathrm{h}} 10^{\mathrm{m}} 12^{\mathrm{s}} .58$, Dec $=+57^{\circ} 43^{\prime} 14.9^{\prime \prime}$ (equinox 2000), with an uncertainty of \pm 6 arcmin (90\% level), independently on each $1 \mathrm{D}$ direction. The preliminary and refined error boxes are shown in Fig. 2.

GRB 070724B also triggered the Konus-Wind (Golenetskii et al. 2007), Suzaku-WAM (Endo et al. 2007) and INTEGRAL SPI-ACS ${ }^{1}$ (V. Beckmann, private communication) experiments, all participating in the InterPlanetary Network (IPN, e.g.

\footnotetext{
${ }^{1}$ http://isdc.unige.ch/cgi-bin/cgiwrap/ beck/ibas/ spiacs/ibas_acs_web.cgi
}
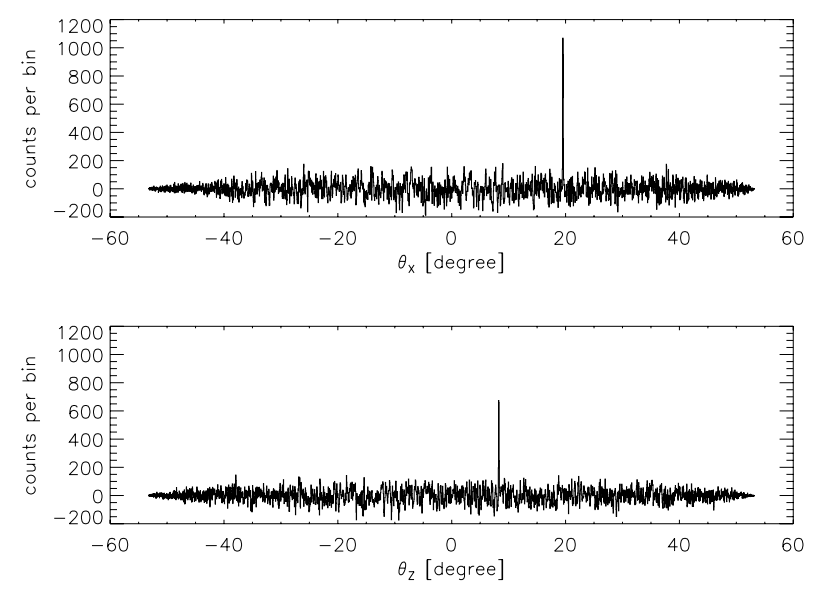

Fig. 1. SuperAGILE images of GRB 070724B. The offset angles $\theta_{X}$ and $\theta_{Z}$ are refer to the satellite reference frame, with a boresight pointing at $\mathrm{RA}=01^{\mathrm{h}} 17^{\mathrm{m}} 30^{\prime} \cdot 44^{\mathrm{s}}$, Dec $=+36^{\circ} 38^{\prime} 45.7^{\prime \prime}$ (equinox 2000).

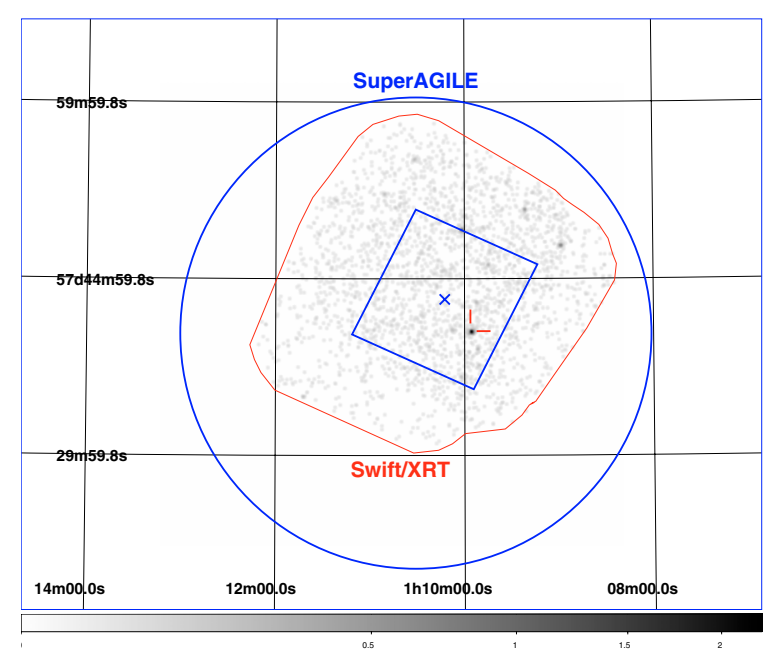

Fig. 2. Swift/XRT image of the field of GRB 070724B, obtained from the total $\sim 16.5 \mathrm{ks}$ PC mode data. The (red) polygon highlights the shape of the sky covered by XRT, obtained with several pointings. Also shown (red marks) is the XRT position, at $\mathrm{RA}(\mathrm{J} 2000)=01^{\mathrm{h}} 09^{\mathrm{m}} 56^{\mathrm{s}} .33$, $\operatorname{Dec}(\mathrm{J} 2000)=+57^{\circ} 40^{\prime} 33^{\prime \prime} 0$ (90\% c.l. error radius of 3'.9). The (blue) circle is the early $20^{\prime}$ SuperAGILE error circle, while the (blue) box inside the XRT region is the SuperAGILE refined $6^{\prime} \times 6^{\prime}$ error box. The cross marks the SuperAGILE refined position.

Hurley et al. 1999). Our GRB localization is consistent with the IPN annulus (K. Hurley \& V. Pal'shin, private communication).

The detection of GRB 070724B is significant up to about $60 \mathrm{keV}$ energy. The GRB duration is $\sim 45 \mathrm{~s}$, with $T_{90}=(40 \pm 1)$ $\mathrm{s}$ in the 20-60 keV energy band. The SuperAGILE light curve of the prompt emission in the same energy band (Fig. 3) shows a multi-peaked structure with four main peaks and internal variability. Considering the fraction of the SuperAGILE detector illuminated by the event, the dead time correction to the observed counts is smaller than $1 \%$.

The hardness ratio, estimated as the ratio of the counts in the $26-60 \mathrm{keV}$ and $20-26 \mathrm{keV}$ energy bands, selected in order to have similar statistics, is shown in the lower panel of Fig. 3. For comparison, the hardness ratio of the Crab Nebula using the same energy bands is $\sim 0.6$. The GRB shows hints of spectral variability even in the narrow energy range covered by SuperAGILE. The time-averaged spectrum in the $20-40 \mathrm{keV}$ energy band, as observed by SuperAGILE, can be described by 


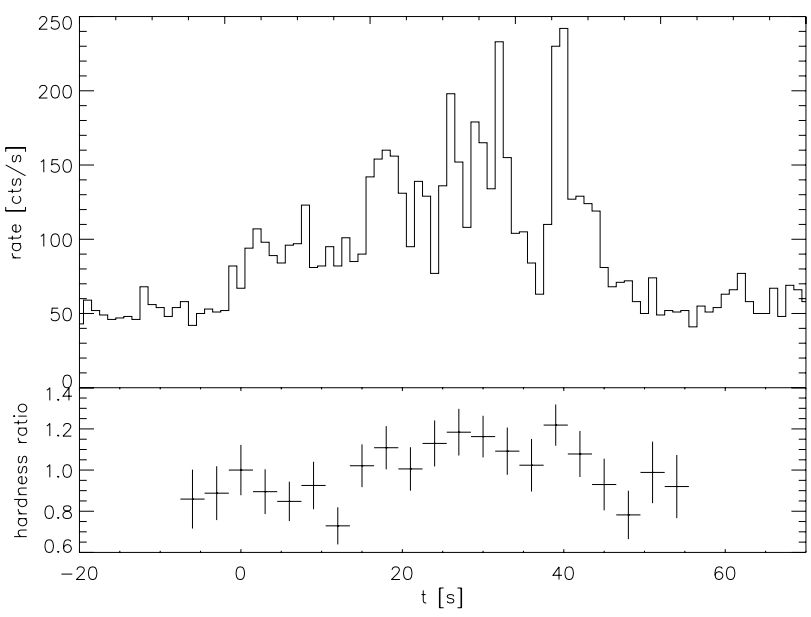

Fig. 3. Upper panel: SuperAGILE lightcurve of GRB 070724B in the $20-60 \mathrm{keV}$ energy band without background subtraction. The start time is 24 July 2007 at 23:25:08 UT. Lower panel: SuperAGILE hardness ratio of GRB $070724 \mathrm{~B}$. The lower energy band is $20-26 \mathrm{keV}$, the higher 26-60 keV, selected in order to have equivalent statistics.

a simple power law with photon index $\Gamma \sim 0.6$. Based on this analysis, the fluence in the $20-40 \mathrm{keV}$ energy band is $\sim 5 \times$ $10^{-6} \mathrm{erg} \mathrm{cm}^{-2}$ and the 1 -s peak flux (assuming the time-averaged spectral shape) is $\sim 4 \times 10^{-7} \mathrm{erg} \mathrm{cm}^{-2} \mathrm{~s}^{-1}$. Uncertainties on these values cannot be taken lower than $50 \%$, due to the current very early calibration status of the SuperAGILE experiment, for offaxis events. The current status of the SuperAGILE response matrix on-axis is such that the spectral parameters and flux of the Crab Nebula are consistent with those from the literature (e.g., Frontera et al. 2007) when a systematic uncertainty of $\sim 15 \%$ is added to the model.

To search for gamma-ray emission from GRB 070724B we analyzed the GRID data as follows. We first applied conservative cuts to the GRID events in order to select only events that could be recognized as celestial gamma-ray photons with a high confidence. We only considered events of reconstructed energy larger than $100 \mathrm{MeV}$ and arrival direction within $5^{\circ}$ from the coordinates of the burst. This resulted in 0 events over a $50 \mathrm{~s}$ long time window starting at $T_{0}$. The lack of events in this time interval is statistically consistent with the expected background rate $(5.1 \times$ $10^{-4}$ cts s$^{-1}$ ) as determined applying the same selection cuts to different time intervals. The largest GRB fluence compatible (at the $99.5 \%$ confidence level) with our observation of 0 photons is $0.02 \mathrm{ph} \mathrm{cm}^{-2}$ with $E>100 \mathrm{MeV}$ (using the preliminary calibrations currently available).

In the $350 \mathrm{keV}-2.8 \mathrm{MeV}$ energy band of the AGILEMinicalorimeter, no statistically significant counting rate increase was found during the $\sim 50 \mathrm{~s}$ of the burst. The corresponding 3- $\sigma$ upper limit is $\sim 4 \times 10^{-6} \mathrm{erg} \mathrm{cm}^{-2}$. This value has been calculated assuming the spectral shape as in Endo et al. (2007) extrapolated to $2.8 \mathrm{MeV}$, and the preliminary effective area obtained by MonteCarlo simulations. The MCAL measurement is consistent with the lack of detectable emission in the KonusWind 300-1160 keV energy band ${ }^{2}$. For this reason this GRB is reminiscent of the BATSE "No High Energy" (NHE) type (Pendleton et al. 1997). The GRB fluence measured by KonusWind is $\left(1.80_{-0.25}^{+0.04}\right) \times 10^{-5} \mathrm{erg} \mathrm{cm}^{-2}(20-500 \mathrm{keV}$ energy band) with a $64-\mathrm{ms}$ peak flux of $\left(2.17_{-0.45}^{+0.34}\right) \times 10^{-6} \mathrm{erg} \mathrm{cm}^{-2} \mathrm{~s}^{-1}$ in the

\footnotetext{
${ }^{2}$ http://www.ioffe.rssi.ru/LEA/GRBs/GRB070724_T84307/
}

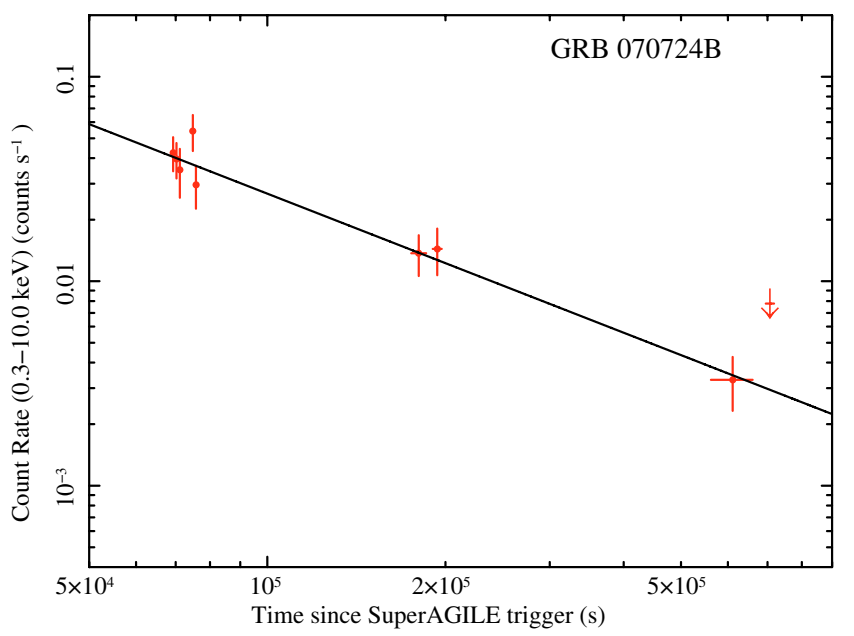

Fig. 4. Swift/XRT light curve of GRB 070724B, corrected for PSF losses, vignetting and background-subtracted. The solid line is the bestfit power-law model $\left(\alpha=1.13_{-0.18}^{+0.23}\right)$.

same energy band and a peak energy of $82 \pm 5 \mathrm{keV}$ (Golenetskii et al. 2007).

\subsection{Afterglow emission}

The Swift data on GRB 070724B were collected as a Target of Opportunity (ToO) observing campaign that commenced on 25 July 2007 at 18:32:39 UT, $19.1 \mathrm{~h}$ after the burst trigger (Romano et al. 2007a). The Swift/XRT data analysis was performed using standard procedures (see, e.g. Romano et al. 2006). The Swift/XRT observations were performed in photoncounting (PC) mode, for a total on-source exposure of $16.5 \mathrm{ks}$. Given the 23. $6 \times 23$. $6 \mathrm{FOV}$, the observations were performed so as to scan most of the area of the $20^{\prime}$-radius SuperAGILE initial error circle. The only decaying source was found at $\mathrm{RA}(\mathrm{J} 2000)=01^{\mathrm{h}} 09^{\mathrm{m}} 56^{\mathrm{s}} .34, \operatorname{Dec}(\mathrm{J} 2000)=+57^{\circ} 40^{\prime} 34^{\prime \prime} .3$, with an uncertainty of 3.'9 (90\% c.l. radius; Romano et al. 2007c). Using the latest version of software and calibration, and combining all available X-ray data, our final position is (Fig. 2) $\mathrm{RA}(\mathrm{J} 2000)=01^{\mathrm{h}} 09^{\mathrm{m}} 56^{\mathrm{s}} .33, \operatorname{Dec}(\mathrm{J} 2000)=+57^{\circ} 40^{\prime} 33^{\prime \prime} .0$, with an error radius of $33^{\prime \prime} 9$. This position is 4.6 from the early SuperAGILE position and 3.5 from the refined one.

The decay and spectral indices are parameterized as $F(v, t) \propto$ $t^{-\alpha} v^{-\beta}$, where $F_{v}\left(\mathrm{erg} \mathrm{cm}^{-2} \mathrm{~s}^{-1} \mathrm{~Hz}^{-1}\right)$ is the monochromatic flux as a function of time $t$ and frequency $v$; we also use $\Gamma=\beta+1$ as the photon index, $N(E) \propto E^{-\Gamma}\left(\mathrm{ph} \mathrm{keV}^{-1} \mathrm{~cm}^{-2} \mathrm{~s}^{-1}\right)$.

Figure 4 shows the XRT light curve in count rate in the $0.3-10 \mathrm{keV}$ energy range, binned so that each point has at least 30 counts, and a $>3-\sigma$ detection is achieved. It exhibits a fading behaviour, with a power-law slope of $\alpha=1.13_{-0.18}^{+0.23}$ $\left(\chi_{\text {red }}^{2}=0.660 / 6\right.$ d.o.f $)$.

We fit the XRT spectrum of the first observation (4 ks) with XSPEC (v11.3.2) using Cash statistics (Cash 1979), which is more appropriate than $\chi^{2}$ statistics given the low number of counts (111), and spectrally unbinned data in the $0.3-10 \mathrm{keV}$ energy range. We adopted an absorbed power law model with free photon index $\Gamma$ and absorption using the photoelectric absorption model tbabs (Wilms et al. 2000) and calculated the goodness of the fit via $10^{4}$ Montecarlo simulations. We obtain $\Gamma=2.4 \pm 0.5$, a column density of $(7 \pm 3) \times 10^{21} \mathrm{~cm}^{-2}$, and a Cash statistic (C-stat) of 371.6 . We find that $76.40 \%$ of the Monte Carlo realizations had fit statistics lower than the observed C-stat. The 
column density is marginally in excess of the Galactic value $\left(N_{\mathrm{H}}^{\mathrm{Gal}}=3.1 \times 10^{21} \mathrm{~cm}^{-2}\right.$; Kalberla et al. 2005). The conversion factor from count rate to unabsorbed flux in the $0.3-10 \mathrm{keV}$ energy range is 1 count $\mathrm{s}^{-1} \sim 1.3 \times 10^{-10} \mathrm{erg} \mathrm{cm}^{-2} \mathrm{~s}^{-1}$, hence the unabsorbed $0.3-10 \mathrm{keV}$ flux for the spectrum at $T+24 \mathrm{~h}$ is $\sim 4 \times$ $10^{-12} \mathrm{erg} \mathrm{cm}^{-2} \mathrm{~s}^{-1}$. This flux estimate places GRB 070724B roughly at the average brightness for Swift GRBs at this epoch of observation.

The Swift/UVOT (Roming et al. 2005) observations covered a $17^{\prime} \times 17^{\prime}$ FOV, which is coaxial with that of the XRT. The UVOT results were reported by (Chester \& Romano 2007). They refer to data collected starting from 2007 Jul. 27 00:02:11 UT, $48.6 \mathrm{~h}$ after the burst trigger time, since the candidate afterglow was outside the UVOT FOV during the first observation. No uncatalogued sources were found in any of the UVOT observations inside the refined Swift/XRT error circle for the candidate afterglow, down to a 3- $\sigma$ limit in the co-added frames of $21.3 \mathrm{mag}$ in the White filter and $19.8 \mathrm{mag}$ in the $V$ filter. These values were not corrected for Galactic extinction, which corresponds to a reddening of $E_{B-V}=0.51 \mathrm{mag}$ in the direction of the burst (Schlegel et al. 1998).

\section{Discussion and conclusions}

GRB 070724B is the first gamma-ray burst localized by the SuperAGILE experiment aboard the AGILE gamma-ray mission, still performing its science verification phase. The field of view of SuperAGILE is co-aligned with, and smaller than, that of the AGILE GRID. Thus, although the SuperAGILE realtime GRB localization rate is generally expected to be a minor addition to what is currently provided (mostly) by Swift/BAT and INTEGRAL/IBIS, any GRB localized by SuperAGILE will necessarily be in the GRID field of view, and will have an associated measurement in the $30 \mathrm{MeV}-50 \mathrm{GeV}$ energy range. This makes any SuperAGILE GRB potentially noticeable, because it may allow us, for the first time, to extend up to the GeV energy band the study of a GRB with an associated multi-wavelength afterglow and distance, measured through its optical redshift. This would be a unique characterization of a GRB, previously impossible. Indeed, for this purpose, AGILE was equipped with on-board triggering and localization capabilities and with a fast communication channel to distribute the few arcmin-level SuperAGILE coordinates worldwide, with anticipated delays of a few minutes. Unfortunately, the on-board procedure was not active at the time of the first event, GRB 070724B, thus the localization was delayed. The fast reaction by Swift allowed us to discover the X-ray afterglow, but the UV, optical and radio searches were carried out with large delays and did not set very tight limits. Last but not least, the AGILE GRID did not detect significant gamma-ray emission during the event. Thus, the distance measurement of an AGILE/GRID burst is postponed until a future opportunity, but GRB 070724B has nonetheless demonstrated us the AGILE capabilities and allowed to observe, for the first time ever, the X-ray afterglow of a GRB with a significant upper limit on its gamma-ray emission.

The emission of photons in the $\sim \mathrm{GeV}$ energy range is required by the commonly accepted GRB emission mechanisms in both external and internal shocks, during the prompt phase (for a review see Mészáros 2006). Recently, the delayed GeV photons in GRB (as in the case of GRB 940217 detected by EGRET) have been theoretically correlated with the delayed X-ray flares detected by Swift (e.g., Burrows et al. 2005b), invoking, for example, the inverse Compton scattering of X-ray flare photons by forward shock electrons (Wang et al. 2006). The afterglow of
GRB 070724B was too weak (due to its late observation) to detect delayed X-ray flares, and indication of time variability in the early X-ray light curve, if any, is just marginal.

The phenomenology of GRBs in the $\sim \mathrm{GeV}$ energy range is still poorly known, and not yet understood, due to the long gap between the demise of the EGRET experiment and the launch of the subsequent gamma-ray mission, AGILE. EGRET imaged high energy photons associated with only five GRBs, also detected by BATSE (Kwok et al. 1993; Hurley et al. 1994; Sommer et al. 1994; Schneid et al. 1995). One other candidate was found by an independent search (Jones et al. 1996). Interestingly, the peak flux of all the five BATSE GRBs was in the high flux tail of the BATSE $\log N-\log S$ distribution, approximately in the top $5 \%$.

GRB 070724B did not show any detectable emission in the gamma ray energy band. Indeed, combining this information with the AGILE/Minicalorimeter and Konus observations (Golenetskii et al. 2007), this event did not show any detectable prompt emission above $300 \mathrm{keV}$, up to $30 \mathrm{GeV}$, and the two properties ("NHE" and lack of gamma emission) may be not unrelated. In order to compare the properties of GRB 070724B with those of the EGRET-detected events, we scaled the 64-ms Konus peak flux to the BATSE standard 50-300 keV energy band and obtained $\sim 9 \mathrm{ph} \mathrm{cm}^{-2} \mathrm{~s}^{-1}\left(\sim 1.4 \times 10^{-6} \mathrm{erg} \mathrm{cm}^{-2} \mathrm{~s}^{-1}\right)^{3}$, thus positioning this event in the brightest $\sim 10 \%$ of the BATSE peak flux distribution. On the other hand, the $50-300 \mathrm{keV}$ fluence of the EGRET-detected GRBs was comparable to that of GRB 070724B $\left(\sim 10^{-5} \mathrm{erg} \mathrm{cm}^{-2} \mathrm{~s}^{-1}\right.$, except for the noticeable case of 940217), but they all had large fluences, also above $300 \mathrm{keV}$, where GRB 070724B was not detected. In particular, the MCAL upper limit is $4 \times 10^{-6} \mathrm{erg} \mathrm{cm}^{-2}$ (in agreement with the measurement in the $0.1-1 \mathrm{MeV}$ energy band by Suzaku, see Endo et al. 2007), a value significantly smaller than the fluence measured by BATSE for the GRBs detected by EGRET. Above $100 \mathrm{MeV}$, our upper limit of $0.02 \mathrm{ph} \mathrm{cm}^{-2}$ on the gamma-ray fluence compares to the fluence $(>100 \mathrm{MeV})$ of the brightest bursts seen with EGRET: $\sim 0.039 \mathrm{ph} \mathrm{cm}^{-2}$ for GRB 930131 (Sommer et al. 1994) and $\sim 0.015 \mathrm{ph} \mathrm{cm}^{-2}$ for GRB 940217 (Hurley et al. 1994).

Thus, our observations and the properties of GRB 070724B are consistent with the scenario suggested by the EGRET and BATSE data, that the prompt gamma-ray emission correlates with the energetics of the prompt emission at high energies (above $300 \mathrm{keV}$ ) and not to those in the "standard" hard X-ray energy range (e.g., 50-300 keV), with only the brightest and hardest GRBs having gamma-ray counterparts above the AGILE (and EGRET) sensitivies. But our observations add a brand new piece of information to this scenario: GRBs with undetectable gamma-ray emission can however be associated with "standard" X-ray afterglow emission, and possibly belong to the class of optically dark events ${ }^{4}$. In fact, an optical afterglow was not detected by the Swift UVOT in the White and $V$ filters, nor by the automated Palomar 60-inch telescope in the $I^{\prime}$ filter, yielding an upper limit of $21.0 \mathrm{mag}$ (Cenko \& Rau 2007) $31.3 \mathrm{~h}$ after the burst. Nor was the afterglow of GRB 070724B detected in the radio band: a search by VLA at a frequency of $8.46 \mathrm{GHz}$ on 5 August 2007 at 10.3 UT ( $\sim 11.5$ days after the event $)$ provided a peak radio brightness of $-25 \pm 36 \mu \mathrm{Jy}$ (Chandra \& Frail 2007).

\footnotetext{
3 Here we assumed the spectral shape as provided by Konus for the time-averaged spectrum. If we assume a power law shape with photon index in the range $1-2$, this number varies from $\sim 7$ to $\sim 12 \mathrm{ph} \mathrm{cm}^{-2} \mathrm{~s}^{-1}$.

4 Adopting the method of the analysis in (De Pasquale et al. 2003), the optical-to-X-ray flux ratio is $f_{\mathrm{oX}} \leq 2 \div 7$.
} 
Of course, the case of GRB 070724B does not allow us to establish a new class of GRBs (yet ...), nor to draw any definite conclusion, but still it allows us to make new correlations between GRB properties, which were previously impossible. Even in the unlikely situation that this will remain a single case, the GRB emission models will need to face these new constraints from now on, in the AGILE era.

Acknowledgements. AGILE is a mission of the Italian Space Agency, with coparticipation of INAF (Istituto Nazionale di Astrofisica) and INFN (Istituto Nazionale di Fisica Nucleare). This work was partially supported by ASI grants $\mathrm{I} / \mathrm{R} / 045 / 04, \mathrm{I} / 089 / 06 / 0, \mathrm{I} / 011 / 07 / 0$ and by the Italian Ministry of University and Research (PRIN 2005025417). INAF personnel at ASDC are under ASI contract I/024/05/1. The authors warmly acknowledge the support by the team of the InterPlanetary Network (IPN).

\section{References}

Burrows, D. N., Hill, J. E., Nousek, J. A., et al. 2005a, Space Sci. Rev., 120, 165 Burrows, D. N., Romano, P., Falcone, A., et al. 2005b, Science, 309, 1833

Cash, W. 1979, ApJ, 228, 939

Cenko, S. B., \& Rau, A. 2007, GRB Coordinates Network, 6679

Chandra, P., \& Frail, D. A 2007, GRB Coordinates Network, 6740

Chen, A., et al. 2007, GRB Coordinates Network, 6670

Chester, M. M., \& Romano, P. 2007, GRB Coordinates Network, 6677

Deckett, M., 1993, in CNES, Small Satellites Systems and Services, 417

Del Monte, E., Costa, E., Di Persio, G., et al. 2007, in Frascati Phys. Ser., Proc.

SciNeGHE07, in press

De Pasquale, M., Piro, L., Perna, R., et al. 2003, ApJ, 592, 1018
Endo, A., Tashiro, M., Urata Y., et al. 2007, GRB Coordinates Network, 6672 Feroci, M., Costa, E., Soffitta, P., et al. 2007a, Nucl. Instr. Meth. A, 581, 728 Feroci, M., Costa, E., Del Monte, E., et al. 2007b, GRB Coordinates Network, 6668

Frontera, F., Orlandini, M., Landi, R., et al. 2007, ApJ, 666, 86

Fuschino, F., Labanti, C., Galli, M., et al. 2007, Proc. Rome Int. Conf. on AstroParticle Physics, Rome 20-22 June 2007, Nucl. Instr. Meth. A, in press Golenetskii, S., Aptekar, R., Mazets, E., et al. 2007, GRB Coordinates Network, 6671

Hurley, K., Dingus, B. L., Mukherjee, R., et al. 1994, Nature, 372, 652

Hurley, K., Briggs, M. S., Kippen, R. M., et al. 1999, ApJS, 120, 399

Jones, B. B., Bertsch, D. L., Dingus, B. L., et al. 1996, ApJ, 463, 565

Kalberla, P. M. W., Burton, W. B., Hartmann, D., et al. 2005, A\&A, 440, 775

Kwok, P. W., et al. 1993, AIP CP, 280, 855

Mészáros, P. 2006, Rep. Prog. Phys., 69, 2259

Pendleton, G. N., Paciesas, W. S., Briggs, M. S., et al. 1997, ApJ, 489, 175

Romano, P., Campana, S., \& Chincarini, G. 2006, A\&A, 456, 917

Romano, P., Kennea, J. A., Guidorzi, C., \& Burrows, D. N. 2007a, GRB Coordinates Network, 6669

Romano, P., Guidorzi, C., Moretti, A., \& Chester, M. M. 2007b, GRB Coordinates Network, 6675

Romano, P., Guidorzi, C., \& Moretti, A. 2007c, GRB Coordinates Network, 6703

Roming, P. W. A., Kennedy, T. E., Mason, K. O., et al. 2005, Space Sci. Rev., 120,95

Schlegel, D. J., Finkbeiner, D. P., \& Davis, M. 1998, ApJ, 500, 525

Schneid, E. J., Bertsch, D. L., Dingus, B. L., et al. 1995, ApJ, 453, 95

Sommer, M., Bertsch, D. L., Dingus, B. L., et al. 1994, ApJ, 422, L63

Tavani, M., Barbiellini, G., Argan, A., et al. 2006, in Proc. SPIE, ed. M. J. L. Turner, \& G. Hasinger, 6266, 626603

Wang, X. Y., Li, Z., \& Mészáros, P. 2006, ApJ, 641, L89

Wilms, J., Allen, A., \& McCray, R. 2000, ApJ, 542, 914 
E. Del Monte et al.: GRB 070724B, Online Material p 1

\section{Online Material}


E. Del Monte et al.: GRB 070724B, Online Material p 2

Table 1. Swift/XRT observation log.

\begin{tabular}{lllll}
\hline \hline Sequence & $\begin{array}{l}\text { Start time (UT) } \\
\text { (yyyy-mm-dd hh:mm:ss) }\end{array}$ & $\begin{array}{l}\text { End time (UT) } \\
\text { (yyyy-mm-dd hh:mm:ss) }\end{array}$ & $\begin{array}{l}\text { Net exposure }^{a} \\
\text { (s) }\end{array}$ & $\begin{array}{l}\text { Time since trigger }^{b} \\
\text { (s) }\end{array}$ \\
\hline 00020055001 & $2007-07-2518: 32: 39$ & $2007-07-2520: 34: 52$ & 3971 & 68852 \\
00020055003 & $2007-07-27$ 00:02:07 & $2007-07-2706: 09: 56$ & 4002 & 175019 \\
00020055004 & $2007-07-3111: 27: 41$ & $2007-07-3117: 57: 58$ & 3139 & 561754 \\
00020055005 & $2007-08-0108: 12: 05$ & $2007-08-0114: 58: 56$ & 3302 & 636418 \\
00020055006 & $2007-08-0200: 31: 49$ & $2007-08-0207: 02: 56$ & 2037 & 695201 \\
\hline
\end{tabular}

${ }^{a}$ The exposure time is spread over several snapshots (single continuous pointings at the target) during each observation.

${ }^{b}$ Start time of the observation in seconds since the SuperAGILE trigger. 